\title{
Solutions to the dynamical equation of polarization-mode dispersion and polarization-dependent losses
}

\author{
Yi Li and Amnon Yariv \\ Department of Applied Physics, California Institute of Technology, Mail Stop 128-95, Pasadena, California 91125
}

Received February 16, 2000; revised manuscript received August 8, 2000

\begin{abstract}
We study the evolution of optical signals in single-mode optical fibers in the presence of polarization-mode dispersion and polarization-dependent losses. Two geometric vectors on the Poincaré sphere are defined to characterize the effects of polarization-mode dispersion and polarization-dependent losses on the optical field in the fiber. By solving the dynamical equation for these two vectors, several general statistical results are obtained. The practically important weak polarization-dependent-loss situation is discussed in detail. (C) 2000 Optical Society of America [S0740-3224(00)02011-7] OCIS codes: $060.4510,260.5430$.
\end{abstract}

\section{INTRODUCTION}

A single-mode optical-communication fiber can support two propagating polarization modes, and these two modes are degenerate if the fiber is isotropic and subject to no external disturbances. In reality, however, this degeneracy is removed by perturbations arising from the irregularities of the fiber core, external stress, temperature fluctuations, etc. Therefore the dispersion characteristics of a propagating mode are, in general, dependent on the state of polarization. Two of the most important polarization-dependent effects are the polarization-mode dispersion (PMD) and the polarization-dependent losses (PDL's). PMD causes polarization-dependent phase delay, whereas PDL causes polarization-dependent attenuation of the propagating optical signal. Both of them are detrimental for optical communications as they cause severe intermodal interferences and lead to pulse broadening and deformation and hence limit the transmission rate of an optical link.

Since these polarization-dependent effects originate largely from random fluctuations along the fiber, their statistical properties are of great importance for assessing their influence on propagating signals and for designing compensation schemes. A rigorous statistical theory of PMD in the absence of PDL was pioneered in 1991 by Foschini and Poole. ${ }^{1}$ In their paper, they proposed a stochastic fiber model from which various statistics of PMD could be obtained by use of the standard methods of stochastical differential equations. Gisin also independently applied similar methods to solve the dynamical equation of PMD. ${ }^{2}$ Following these original studies, the statistics of PMD has received wide and continued interest. $^{3-7}$ These investigations, among others, have established a satisfactory, if not complete, theoretical understanding of the statistical nature of PMD in the absence of PDL.

Despite the triumphant progress in studying the statistics of PMD, a parallel study of the statistics of combined effects of PMD and PDL has long been missing. The first serious investigation was carried out by Gisin and Huttner. $^{8}$ In their study, Gisin and Huttner defined a complex vector, which they referred to as the "principal state vector," to describe optical signal propagation in the presence of both PMD and PDL. They also derived the dynamical equation for the evolution of the principal state vector. Since then, attention has been drawn to the issue of combined effects of PMD and PDL, but most investigations are focused on particular phenomenological effects or collecting empirical statistics. ${ }^{9-11}$ Neither analytic solutions to the dynamical equation nor general statistical relations have been reported to the best of the authors' knowledge. In this paper we present some basic strategies for solving the dynamical equation and glean some general statistical results that may be useful and of interest for future studies.

\section{GEOMETRICAL REPRESENTATION OF POLARIZATION-MODE DISPERSION AND POLARIZATION-DEPENDENT LOSSES}

The optical field in a weakly guided single-mode fiber can be conveniently described by a two-dimensional complex column vector on the basis of two transverse linear polarization modes. ${ }^{12}$ Borrowing Dirac's notation from quantum mechanics, ${ }^{13,14}$ we denote this two-component vector by $|\varphi\rangle$. In the following analysis, $|\varphi\rangle$ is not assumed to be normalized. We treat $|\varphi\rangle$ and $a|\varphi\rangle$ as the same polarization state for any complex multiplier $a$, since the absolute overall phase and intensity are irrelevant to our discussion. We assume that the input optical signal has a fixed polarization state denoted by $\left|\varphi_{0}\right\rangle$. After transmission through a distance in an optical fiber, the polarization state of the optical signal evolves to

$$
|\varphi\rangle=T(\omega)\left|\varphi_{0}\right\rangle \text {. }
$$

Here $T$ is a complex $2 \times 2$ transmission matrix that depends on both the propagating distance $z$ and the optical 
(circular) frequency $\omega$. In the above equation we have suppressed its dependence on $z$ in the argument. For optical fibers with only PMD and PDL effects, $T(\omega)$ is nonsingular and hence can always be normalized as $\operatorname{det} T(\omega)=1$. We remark that in the presence of PDL the transmission matrix $T(\omega)$ is not unitary, in contrast with the pure PMD case. The frequency-domain evolution of the state vector is given by

$$
\frac{\partial}{\partial \omega}|\varphi\rangle=T_{\omega} T^{-1}|\varphi\rangle \text {. }
$$

It can also be shown that the matrix $T_{\omega} T^{-1}$ is traceless. ${ }^{8}$ Consequently, it can be uniquely decomposed into a linear superposition of the three Pauli matrices over the field of complex numbers:

$$
T_{\omega} T^{-1}=-\frac{i}{2} \mathbf{W} \cdot \boldsymbol{\sigma},
$$

where $\boldsymbol{\sigma}$ is the Pauli matrix vector whose three components are

$$
\sigma_{1}=\left[\begin{array}{ll}
0 & 1 \\
1 & 0
\end{array}\right], \quad \sigma_{2}=\left[\begin{array}{cc}
0 & -i \\
i & 0
\end{array}\right], \quad \sigma_{3}=\left[\begin{array}{cc}
1 & 0 \\
0 & -1
\end{array}\right] .
$$

The complex vector $\mathbf{W}$ in Eq. (3) is the principal state vector originally defined by Gisin and Huttner, and it obeys the following dynamical equation ${ }^{8}$ :

$$
\frac{\partial \mathbf{W}}{\partial z}=\boldsymbol{\beta}(z)+[\omega \boldsymbol{\beta}(z)+i \boldsymbol{\alpha}(z)] \times \mathbf{W},
$$

where $z$ is the propagation distance along the optical fiber, $\boldsymbol{\beta}$ is the local birefringence vector, and $\boldsymbol{\alpha}$ is the local PDL vector.

Before embarking on solving the dynamical equation, it is instructive to take a look at the geometric interpretation of $\mathbf{W}$. Denoting its real and imaginary parts by $\boldsymbol{\Omega}$ and $\Lambda$, we have

$$
\frac{\partial}{\partial \omega}|\varphi\rangle=-\frac{i}{2}(\boldsymbol{\Omega}+i \boldsymbol{\Lambda}) \cdot \boldsymbol{\sigma}|\varphi\rangle .
$$

The evolution of the complex state vector $|\varphi\rangle$ is more intuitively represented in the three-dimensional Euclidean space by the Stoke's vector, which is defined as

$$
\mathbf{S} \equiv \frac{\langle\varphi|\boldsymbol{\sigma}| \varphi\rangle}{\langle\varphi \mid \varphi\rangle}
$$

Since $\boldsymbol{\sigma}$ is Hermitian, it follows that $\mathbf{S}$ is real. It can also be shown that $\mathbf{S}$ is of unit length; thus its end point moves on the surface of a unit sphere as $\omega$ changes. This representation of the state vector $|\varphi\rangle$ is often termed as the Poincaré-sphere representation. Taking the frequency derivative of Eq. (7) and making use of the state-evolution equation (6), we obtain the generalized PMD equation:

$$
\frac{\partial \mathbf{S}}{\partial \omega}=\mathbf{\Omega} \times \mathbf{S}-(\boldsymbol{\Lambda} \times \mathbf{S}) \times \mathbf{S} .
$$

In arriving at Eq. (8) we have used the following property of the Pauli matrices:

$$
\sigma_{m} \sigma_{n}=\delta_{m n} I+i \sum_{l=1}^{3} \epsilon_{m n l} \sigma_{l},
$$

where $I$ is the $2 \times 2$ unit matrix and $\epsilon_{m n l}$ is the LeviCivita pseudotensor. ${ }^{15}$ A similar result is obtained by Eyal $^{16}$ in a study of PMD in nonunitary systems. The approach shown above is different and, in addition, establishes the connection between the generalized PMD equation and the dynamical equation of the principal state vector. The importance of this connection is that, once the solutions to the dynamical equation are obtained, they can be readily used to describe the influence of PMD and PDL on the propagation of signals by means of generalized PMD equation (8).

From the geometric point of view, Eq. (8) can be regarded as an alternative definition of $\boldsymbol{\Omega}$ and $\boldsymbol{\Lambda}$. In the absence of PMD, generalized PMD equation (8) reduces to Poole's PMD equation:

$$
\partial \mathbf{S} / \partial \omega=\mathbf{\Omega}_{\mathrm{PMD}} \times \mathbf{S},
$$

where $\boldsymbol{\Omega}_{\mathrm{PMD}}$ is the conventionally defined PMD vector. In this limiting case of pure PMD, $\boldsymbol{\Lambda}$ vanishes and $\boldsymbol{\Omega}$ reduces to $\boldsymbol{\Omega}_{\mathrm{PMD}}$. The Poole-Wagner principal states of polarization ${ }^{17}$ can also be extended to more general cases that involve both PMD and PDL. The generalized principal states of polarization are defined to be the polarization states that are frequency independent to first order: $\partial \mathbf{S}_{p} / \partial \omega=0$. By Eq. (8) this condition is equivalent to solving the following algebraic eigenvalue problem:

$$
\boldsymbol{\Omega}-\boldsymbol{\Lambda} \times \mathbf{S}_{p}=\lambda \mathbf{S}_{p},
$$

where $\lambda$ is a real eigenvalue to be determined. Together with the identity $\mathbf{S}_{p}^{2}=1$, the eigenvalue problem can be solved for the generalized principal states of polarization $\mathbf{S}_{p}$, although the calculation is more involved than in the case of pure PMD.

\section{SOLUTIONS OF THE DYNAMICAL EQUATION}

Since $\boldsymbol{\Omega}$ and $\boldsymbol{\Lambda}$ completely determine the evolution of the Stokes vector, the solutions of the dynamical equation (5) will provide detailed information about the combined effects of PMD and PDL on propagating optical signals. Therefore we shall concentrate on solving the dynamical equation for the rest of this paper. Expressed in $\boldsymbol{\Omega}$ and $\Lambda$, Eq. (5) becomes

$$
\begin{aligned}
& \frac{\partial \boldsymbol{\Omega}}{\partial z}=\boldsymbol{\beta}+\omega \boldsymbol{\beta} \times \boldsymbol{\Omega}-\boldsymbol{\alpha} \times \boldsymbol{\Lambda}, \\
& \frac{\partial \boldsymbol{\Lambda}}{\partial z}=\omega \boldsymbol{\beta} \times \boldsymbol{\Lambda}+\boldsymbol{\alpha} \times \boldsymbol{\Omega} .
\end{aligned}
$$

Before solving these equations, it is necessary to specify the stochastic nature of $\boldsymbol{\alpha}$ and $\boldsymbol{\beta}$. The first assumption we make is that $\boldsymbol{\alpha} \| \boldsymbol{\beta}$. The same assumption is also adopted by Gisin and Huttner. ${ }^{8}$ Although it is an idealization of the real situation, this assumption still captures the essence of the problem while greatly simplifying theoretical calculations. In a real fiber the random local birefringence and differential losses usually arise 
from the same perturbations. Consequently some correlation between $\boldsymbol{\alpha}$ and $\boldsymbol{\beta}$ is expected. The assumption that $\boldsymbol{\alpha} \| \boldsymbol{\beta}$ can be regarded as an extreme case of this correlation. Secondly, we assume $\boldsymbol{\alpha}$ and $\boldsymbol{\beta}$ to be linear. In the Poincaré-sphere representation this means that $\boldsymbol{\beta}$ and $\boldsymbol{\alpha}$ lie on the great circle of linear polarization. Their magnitudes, $\beta$ and $\alpha$, are taken to be constant, whereas their azimuthal angle in the plane of linear polarization, $\theta(z)$, is modeled as a Wiener process ${ }^{18}$ :

$$
\left\langle\xi_{z}\right\rangle=0, \quad\left\langle\xi_{z} \xi_{z^{\prime}}\right\rangle=\frac{2}{h} \delta\left(z-z^{\prime}\right), \quad \xi_{z} \equiv \mathrm{d} \theta / \mathrm{d} z .
$$

Here $h$ is the autocorrelation length of the fiber, and the angled brackets are used to denote the ensemble average. This model is similar to that used by Wai and Menyuk in Ref. 3.

Dynamical equation (12) can be simplified by switching to a reference frame that rigidly rotates with $\boldsymbol{\beta}$ (and hence $\boldsymbol{\alpha})$. The statistics of scalar quantities, such as $\boldsymbol{\Omega}^{2}$ and $\boldsymbol{\Omega} \cdot \boldsymbol{\Lambda}$, are not affected by this changing of coordinate systems, since scalars are invariants of coordinate transformations. Specifically, axis 1 of the rotating coordinates is chosen to be aligned with $\boldsymbol{\beta}$ and axis 3 with the direction of signal propagation. The same rotating reference frame was adopted by Foschini and Poole ${ }^{1}$ and by Wai and Menyuk ${ }^{3}$ in their study on PMD statistics in the absence of PDL. In this coordinate system the dynamical equation, written in the six components of $\boldsymbol{\Omega}$ and $\boldsymbol{\Lambda}$, reads

$$
\frac{\partial}{\partial z}\left[\begin{array}{c}
\Omega_{1} \\
\Omega_{2} \\
\Omega_{3} \\
\Lambda_{1} \\
\Lambda_{2} \\
\Lambda_{3}
\end{array}\right]=\left[\begin{array}{c}
\Omega_{2} \\
-\Omega_{1} \\
0 \\
\Lambda_{2} \\
-\Lambda_{1} \\
0
\end{array}\right] \xi_{z}+\left[\begin{array}{c}
\beta \\
-\omega \beta \Omega_{3}+\alpha \Lambda_{3} \\
\omega \beta \Omega_{2}-\alpha \Lambda_{2} \\
0 \\
-\alpha \Omega_{3}-\omega \beta \Lambda_{3} \\
\alpha \Omega_{2}+\omega \beta \Lambda_{2}
\end{array}\right]
$$

It should be noted that all the components of $\boldsymbol{\Omega}$ and $\boldsymbol{\Lambda}$ in the above equation are now measured in the rotating coordinate system.

Owing to the stochastic nature of $\xi_{z}$, the dynamical equation (14) is a six-dimensional stochastical differential equation (SDE). A systematic way to solve a SDE is to construct a linear differential operator called the generator. ${ }^{19,20}$ Once the generator $\hat{G}$ for the dynamical equation is constructed, the expectation value of an arbitrary well-behaved function of $\boldsymbol{\Omega}$ and $\boldsymbol{\Lambda}$, say, $f(\boldsymbol{\Omega}, \boldsymbol{\Lambda})$, obeys the following moment equation:

$$
\frac{\partial}{\partial z}\langle f\rangle=\langle\hat{G} f\rangle
$$

Usually Eq. (15) is not closed by itself. New unknowns are often contained in $\langle\hat{G} f\rangle$. However, it is sometimes possible to find a closed set of first-order equations by repeatedly applying Eq. (15) to each unknown. That way the desired quantity $\langle f\rangle$ can be determined in principle.

By the theory of stochastic differential equations, a given SDE admits two different interpretations: the Itô interpretation and the Stratonovich interpretation. ${ }^{19}$ These two interpretations naturally lead to different generators. Depending on the physical nature of the prob- lem at hand, one needs to choose the appropriate representation. In our case it has been shown that the appropriate interpretation to adopt is the Stratonovich interpretation. ${ }^{18,1,3}$ A discussion on constructing the Stratonovich generator of a SDE of the type of Eq. (14) can be found in standard reference books ${ }^{18-20}$ and shall not be repeated here. Here we merely quote the resulting Stratonovich generator for stochastic dynamical equation (14):

$$
\begin{aligned}
\hat{G}= & \beta \frac{\partial}{\partial \Omega_{1}}-\left(\omega \beta \Omega_{3}-\alpha \Lambda_{3}\right) \frac{\partial}{\partial \Omega_{2}}+\left(\omega \beta \Omega_{2}-\alpha \Lambda_{2}\right) \frac{\partial}{\partial \Omega_{3}} \\
& -\left(\alpha \Omega_{3}+\omega \beta \Lambda_{3}\right) \frac{\partial}{\partial \Lambda_{2}}+\left(\alpha \Omega_{2}+\omega \beta \Lambda_{2}\right) \frac{\partial}{\partial \Lambda_{3}} \\
& +\frac{1}{h}\left(\Omega_{2}{ }^{2} \frac{\partial^{2}}{\partial \Omega_{1}{ }^{2}}+\Omega_{1}{ }^{2} \frac{\partial^{2}}{\partial \Omega_{2}{ }^{2}}+\Lambda_{2}{ }^{2} \frac{\partial^{2}}{\partial \Lambda_{1}{ }^{2}}+\Lambda_{1}{ }^{2} \frac{\partial^{2}}{\partial \Lambda_{2}{ }^{2}}\right. \\
& -2 \Omega_{1} \Omega_{2} \frac{\partial^{2}}{\partial \Omega_{1} \partial \Omega_{2}}+2 \Omega_{2} \Lambda_{2} \frac{\partial^{2}}{\partial \Omega_{1} \partial \Lambda_{1}} \\
& -2 \Omega_{2} \Lambda_{1} \frac{\partial^{2}}{\partial \Omega_{1} \partial \Lambda_{2}}-2 \Omega_{1} \Lambda_{2} \frac{\partial^{2}}{\partial \Omega_{2} \partial \Lambda_{1}} \\
& +2 \Omega_{1} \Lambda_{1} \frac{\partial^{2}}{\partial \Omega_{2} \partial \Lambda_{2}}-2 \Lambda_{1} \Lambda_{2} \frac{\partial^{2}}{\partial \Lambda_{1} \partial \Lambda_{2}} \\
& \left.-\Omega_{1} \frac{\partial}{\partial \Omega_{1}}-\Omega_{2} \frac{\partial}{\partial \Omega_{2}}-\Lambda_{1} \frac{\partial}{\partial \Lambda_{1}}-\Lambda_{2} \frac{\partial}{\partial \Lambda_{2}}\right) .
\end{aligned}
$$

With this specific form of $\hat{G}$ we can start calculating various scalar expectation values. First we calculate $\langle\boldsymbol{\Omega} \cdot \boldsymbol{\Lambda}\rangle$. By moment equation (15) we have

$$
\frac{\partial}{\partial z}(\boldsymbol{\Omega} \cdot \boldsymbol{\Lambda}\rangle=\frac{\partial}{\partial z}\left\langle\Omega_{1} \Lambda_{1}+\Omega_{2} \Lambda_{2}+\Omega_{3} \Lambda_{3}\right\rangle=\beta\left\langle\Lambda_{1}\right\rangle .
$$

To close Eq. (17), we need to evaluate a new unknown, $\left\langle\Lambda_{1}\right\rangle$. The moment equation for $\Gamma_{1}$ is

$$
\frac{\partial}{\partial z}\left\langle\Lambda_{1}\right\rangle=-\frac{1}{h}\left\langle\Lambda_{1}\right\rangle
$$

The only solution to Eq. (18) satisfying initial condition $\left\langle\Lambda_{1}\right\rangle_{z=0}=0$ is $\left\langle\Lambda_{1}\right\rangle=0$. Therefore the solution to Eq. (17) is

$$
\langle\mathbf{\Omega} \cdot \boldsymbol{\Lambda}\rangle=\mathbf{0} .
$$

Equation (19) implies that, on average, $\boldsymbol{\Omega}$ and $\boldsymbol{\Lambda}$ are perpendicular to each other. This does not mean that $\boldsymbol{\Omega}$ and $\Lambda$ are mutually orthogonal. In fact they are not, since, as we show below, $(\boldsymbol{\Omega} \cdot \boldsymbol{\Lambda})^{2}$ has a nonvanishing average value.

Next, we calculate $\left\langle\boldsymbol{\Omega}^{2}\right\rangle$ and $\left\langle\boldsymbol{\Lambda}^{2}\right\rangle$ :

$$
\begin{aligned}
& \frac{\partial}{\partial z}\langle\boldsymbol{\Omega}\rangle^{2}=\frac{\partial}{\partial z}\left(\Omega_{1}^{2}+\Omega_{2}^{2}+\Omega_{3}^{2}\right\rangle=2 \beta\left\langle\Omega_{1}\right\rangle+2 \alpha g, \\
& \frac{\partial}{\partial z}\left\langle\Lambda^{2}\right)=\frac{\partial}{\partial z}\left\langle\Lambda_{1}^{2}+\Lambda_{2}^{2}+\Lambda_{3}^{2}\right\rangle=2 \alpha g,
\end{aligned}
$$


where $g \equiv\left\langle\Omega_{2} \Lambda_{3}-\Omega_{3} \Lambda_{2}\right\rangle$. It follows that

$$
\frac{\partial}{\partial z}\left\langle\mathbf{\Omega}^{2}-\Lambda^{2}\right\rangle=2 \beta\left\langle\Omega_{1}\right\rangle \text {. }
$$

To solve it we need to calculate $\left\langle\Omega_{1}\right\rangle$, which satisfies

$$
\frac{\partial}{\partial z}\left\langle\Omega_{1}\right\rangle=\beta-\frac{1}{h}\left\langle\Omega_{1}\right\rangle .
$$

The solution to Eq. (23) with initial condition $\left\langle\Omega_{1}\right\rangle_{z=0}$ $=0$ is

$$
\left\langle\Omega_{1}\right\rangle=\beta h[1-\exp (-z / h)]
$$

and it follows that

$$
\left\langle\boldsymbol{\Omega}^{2}-\boldsymbol{\Lambda}^{2}\right\rangle=2 \beta^{2} h^{2}\left[\frac{z}{h}-1+\exp (-z / h)\right] .
$$

It is interesting to compare Eq. (25) with the wellknown solution for the mean-square PMD in the absence of $\mathrm{PDL}^{1,3}$ :

$$
\left\langle\mathbf{\Omega}^{2}\right\rangle_{\mathrm{PMD}}=2 \beta^{2} h^{2}\left[\frac{z}{h}-1+\exp (-z / h)\right] .
$$

It is remarkable that the solutions of $\left\langle\boldsymbol{\Omega}^{2}-\boldsymbol{\Lambda}^{2}\right\rangle$ and $\left\langle\boldsymbol{\Omega}^{2}\right\rangle_{\mathrm{PMD}}$ are identical. Equation (25) incorporates the familiar pure PMD relation (26) as a special case of $\Lambda$ $\rightarrow \mathbf{0}$. In particular we note that when $z \gg h,\left\langle\mathbf{\Omega}^{2}\right.$ $\left.-\Lambda^{2}\right\rangle \simeq 2 \beta^{2} h z$, a well-known result for $\left\langle\Omega^{2}\right\rangle_{\mathrm{PMD}}$ in the long-length regime.

Although $\left\langle\boldsymbol{\Omega}^{2}-\boldsymbol{\Lambda}^{2}\right\rangle$ is found with little effort, the expectation values of $\Omega^{2}$ and $\Lambda^{2}$ are more difficult to obtain. To close Eqs. (20) and (21), we have the following moment equation for $g$ :

$$
\frac{\partial}{\partial z} g=-\frac{1}{h} g+\alpha\left\langle\Omega_{2}^{2}+\Omega_{3}^{2}+\Lambda_{2}^{2}+\Lambda_{3}^{2}\right\rangle .
$$

This in turn gives us a new unknown, $\left\langle\Omega_{2}^{2}+\Omega_{3}^{2}+\Lambda_{2}^{2}\right.$ $\left.+\Lambda_{3}^{2}\right\rangle$. It turns out that repeatedly applying moment equation (15) to each new unknown leads to a large set of coupled differential equations whose precise analytic solutions are not very illuminating if not totally intractable. Therefore we seek approximate solutions for the practically important situation of long-range $(z \gg h)$ and weak PDL $(\alpha h \ll 1)$. These conditions hold for most communication fibers. In this regime we postulate the following quasi-spherical symmetry:

$$
\left\langle\Omega_{1}^{2}\right\rangle \simeq\left\langle\Omega_{2}^{2}\right\rangle \simeq\left\langle\Omega_{3}^{2}\right\rangle, \quad\left\langle\Lambda_{1}^{2}\right\rangle \simeq\left\langle\Lambda_{2}^{2}\right\rangle \simeq\left\langle\Lambda_{3}^{2}\right\rangle .
$$

We note that spherical symmetry is a natural assumption to make in the lab's reference frame; however, it is not so in the rotating frame that we adopt for our calculation. Indeed, as shown in Section 5, perfect spherical symmetry does not exist in this rotating reference frame. Nevertheless, as we explain in detail in Section 5, Eqs. (28) still serve as a good approximation under the weak PDL assumption. Inserting Eqs. (28) into Eq. (27) yields

$$
\frac{\partial}{\partial z} g \simeq-\frac{1}{h} g+\frac{2 \alpha}{3}\left\langle\mathbf{\Omega}^{2}+\Lambda^{2}\right\rangle .
$$

From Eqs. (20), (21), (24), and (29) we obtain the following linear second-order differential equation for $g$ :

$$
\frac{\mathrm{d}^{2} g}{\mathrm{~d} z^{2}}+\frac{1}{h} \frac{\mathrm{dg}}{\mathrm{d} z}-\frac{8 \alpha^{2}}{3} g=\frac{4}{3} \beta^{2} \alpha h[1-\exp (-z / h)] .
$$

With initial conditions $g_{z=0}=g_{z=0}^{\prime}=0$ and the weak PDL condition $\alpha h \ll 1$, the asymptotic solution to Eq. (30) for $z \gg h$ is found to be

$$
g=\left\langle\Omega_{2} \Lambda_{3}-\Omega_{3} \Lambda_{2}\right\rangle \simeq \frac{\beta^{2} h}{2 \alpha}[\exp (z / \Gamma)-1],
$$

where

$$
\Gamma \equiv\left(\frac{8 \alpha^{2} h}{3}\right)^{-1}
$$

Now Eqs. (20) and (21) can be integrated to give

$$
\begin{aligned}
& \left\langle\boldsymbol{\Omega}^{2}\right\rangle \simeq \beta^{2} h \Gamma\left[\exp (z / \Gamma)-1+\frac{z}{\Gamma}\right], \\
& \left\langle\Lambda^{2}\right\rangle \simeq \beta^{2} h \Gamma\left[\exp (z / \Gamma)-1-\frac{z}{\Gamma}\right] .
\end{aligned}
$$

If $z \ll \Gamma$, then $\left\langle\boldsymbol{\Omega}^{2}\right\rangle \simeq 2 \beta^{2} h z$ is approximately the same as $\left\langle\boldsymbol{\Omega}^{2}\right\rangle_{\mathrm{PMD}}$ of the pure PMD case. When $z$ approaches $\Gamma$, the statistics start to deviate significantly from the statistics of the pure PMD case. Therefore $\Gamma$ characterizes the distance at which the mixed effects of PMD and PDL become prominent. Under the weak PDL assumption it is obvious that $\Gamma \gg h$. For a rough estimate, take $h$ $\sim 40 \mathrm{~m}, \alpha \sim 10^{-4}$, and then $\Gamma \sim 10^{6} \mathrm{~m}$. Since $\Gamma$ is usually many orders of magnitude larger than $h$, there is a wide range of $z$ that satisfies $h \ll z \ll \Gamma$. In this length regime, $\left\langle\boldsymbol{\Omega}^{2}\right\rangle$ scales linearly and $\left\langle\boldsymbol{\Lambda}^{2}\right\rangle$ scales quadratically in $z$, as can be easily seen from Eqs. (33) and (34). In the regime of $z \gg \Gamma$, which is rarely the case for opticalcommunication fibers, both grow exponentially in $z$.

Finally we calculate $\left\langle(\boldsymbol{\Omega} \cdot \boldsymbol{\Lambda})^{2}\right\rangle$. The moment equation reads

$$
\frac{\partial}{\partial z}\left\langle(\boldsymbol{\Omega} \cdot \boldsymbol{\Lambda})^{2}\right\rangle=2 \beta\left\langle\Omega_{1} \Lambda_{1}^{2}+\Omega_{2} \Lambda_{1} \Lambda_{2}+\Omega_{3} \Lambda_{1} \Lambda_{3}\right\rangle .
$$

Again, precise analytic solution of Eq. (35) would require tackling a big set of coupled differential equations, so we seek an approximate solution. First we note that $\left\langle\Omega_{2} \Lambda_{1} \Lambda_{2}\right\rangle$ and $\left\langle\Omega_{3} \Lambda_{1} \Lambda_{3}\right\rangle$ are expected to contribute much less than $\left\langle\Omega_{1} \Lambda_{1}^{2}\right\rangle$ since the product of different $\Lambda$ components tends to average to zero. Therefore we can neglect them in a leading-order approximation. We make a further simplification by replacing $\left\langle\Omega_{1} \Lambda_{1}^{2}\right\rangle$ with $\left\langle\Omega_{1}\right\rangle\left\langle\Lambda_{1}^{2}\right\rangle$, since the correlation between $\Omega_{1}$ and $\Lambda_{1}^{2}$ is expected to be weak. After all these approximations, Eq. (35) reduces to

$$
\frac{\partial}{\partial z}\left\langle(\boldsymbol{\Omega} \cdot \boldsymbol{\Lambda})^{2}\right\rangle \simeq 2 \beta\left\langle\Omega_{1}\right\rangle\left\langle\Lambda_{1}^{2}\right\rangle \simeq 2 \beta^{2} h \frac{\left\langle\Lambda^{2}\right\rangle}{3} .
$$

Since $\left\langle\Lambda^{2}\right\rangle$ has already been obtained, Eq. (36) can now be integrated to give 


$$
\left\langle(\boldsymbol{\Omega} \cdot \boldsymbol{\Lambda})^{2}\right\rangle \simeq \frac{2 \beta^{4} h^{2} \Gamma^{2}}{3}\left[\exp (z / \Gamma)-1-\frac{z}{\Gamma}-\frac{z^{2}}{2 \Gamma^{2}}\right] .
$$

In the length regime of $h \ll z \ll \Gamma$, we have $\left\langle(\boldsymbol{\Omega} \cdot \boldsymbol{\Lambda})^{2}\right\rangle$ $\sim z^{3}$.

\section{NUMERICAL SIMULATION}

To confirm the validity of the model and various approximations made during the calculation, numerical simulations are performed to verify the statistic results for $\left\langle\boldsymbol{\Omega}^{2}\right\rangle,\left\langle\boldsymbol{\Lambda}^{2}\right\rangle,\langle\boldsymbol{\Omega} \cdot \boldsymbol{\Lambda}\rangle$, and $\left\langle(\boldsymbol{\Omega} \cdot \boldsymbol{\Lambda})^{2}\right\rangle$. Fibers with lengths up to $1000 \mathrm{~km}$ are simulated. The autocorrelation length is taken to be $20 \mathrm{~m}$, and the characteristic length $\Gamma$ is $1875 \mathrm{~km}$, corresponding to $\alpha=10^{-4}$. The statistical data are collected from 1000 randomly generated fibers. The solid curves in Figs. 1-3 show the ensemble average predicted by the theory, and discrete circles and triangles are data from numerical calculations. The agreement is excellent.

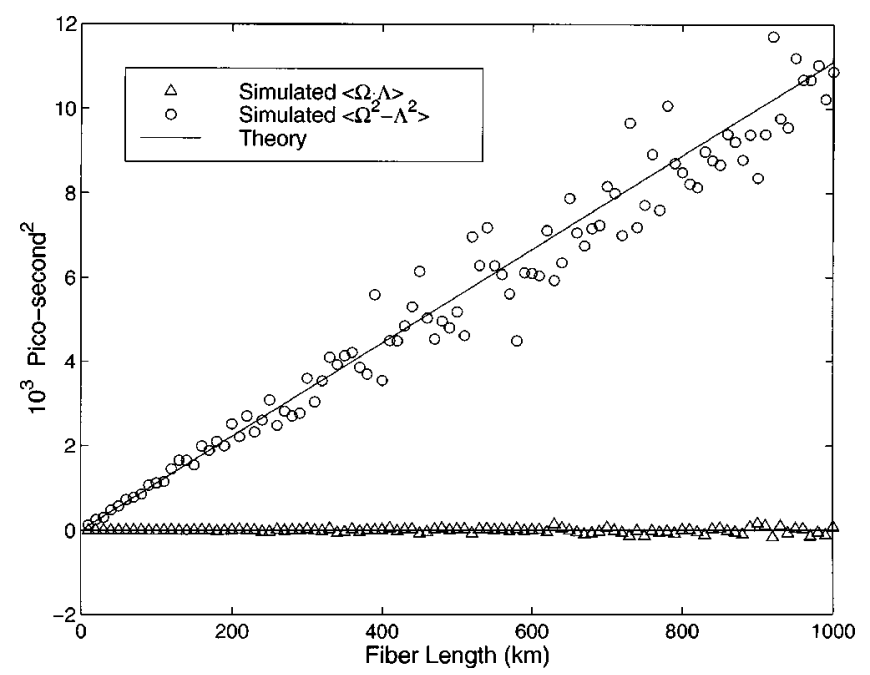

Fig. 1. Ensemble average of $\boldsymbol{\Omega} \cdot \boldsymbol{\Lambda}$ and $\boldsymbol{\Omega}^{2}-\boldsymbol{\Lambda}^{2}$.

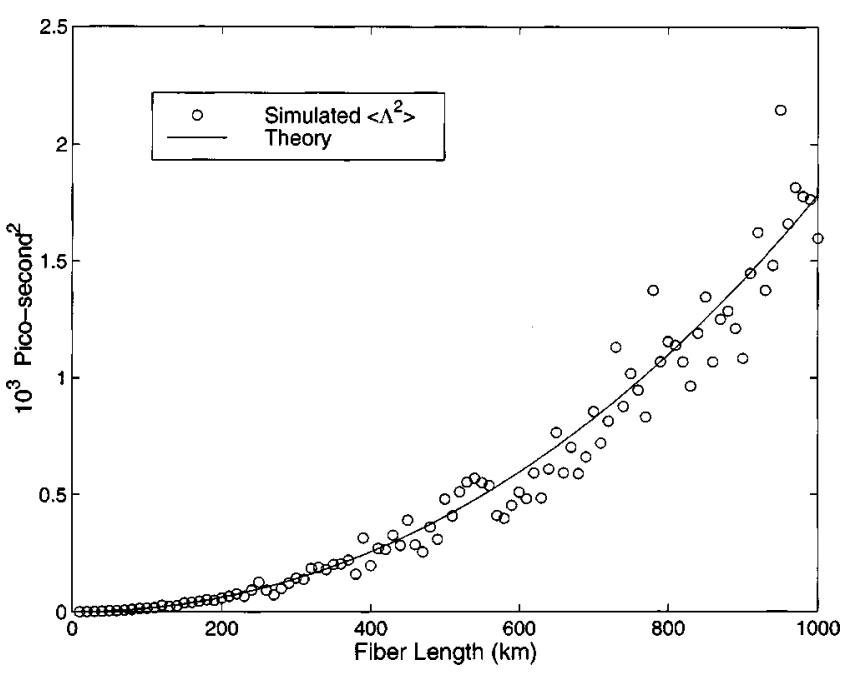

Fig. 2. Ensemble average of $\Lambda^{2}$.

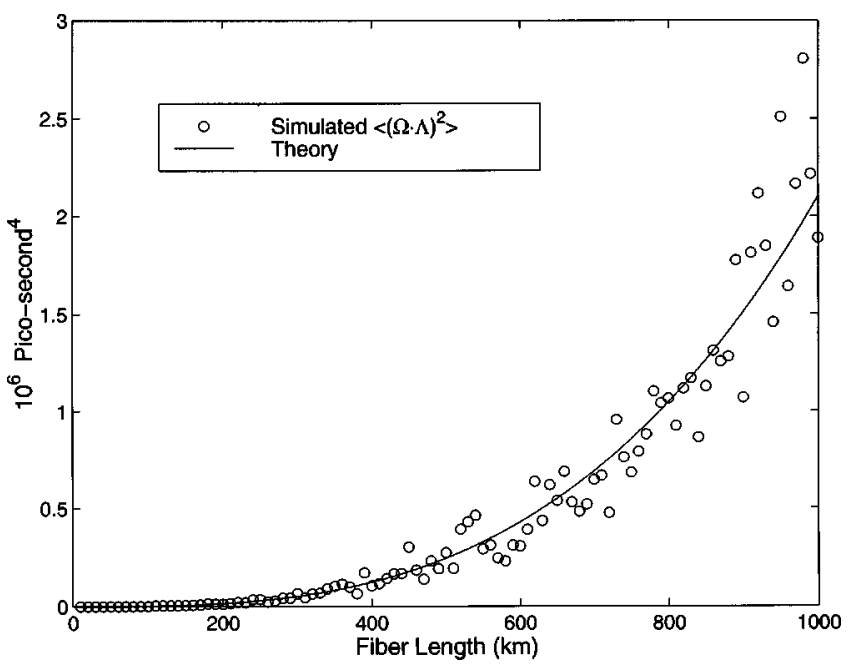

Fig. 3. Ensemble average of $(\boldsymbol{\Omega} \cdot \boldsymbol{\Lambda})^{2}$.

\section{DISCUSSION AND EXAMPLE}

We now justify the hypothesis, Eq. (28), made earlier in our calculation. It suffices to show that $\left|\left\langle\Omega_{i}^{2}\right\rangle-\left\langle\Omega_{j}^{2}\right\rangle\right|$ $\ll\left\langle\Omega_{i}^{2}\right\rangle$ and $\left|\left\langle\Lambda_{i}^{2}\right\rangle-\left\langle\Lambda_{j}^{2}\right\rangle\right| \ll\left\langle\Lambda_{i}^{2}\right\rangle$ for $i \neq j$. To illustrate the idea, we consider one simple example. The moment equation for $\Lambda_{1}^{2}$ states

$$
\frac{\partial}{\partial z}\left\langle\Lambda_{1}^{2}\right\rangle=\frac{2}{h}\left\langle\Lambda_{2}^{2}-\Lambda_{1}^{2}\right\rangle .
$$

We note immediately that perfect spherical symmetry does not hold in the rotating reference frame, since otherwise Eq. (38) would imply that $\left\langle\Lambda_{1}^{2}\right\rangle$ is constant. This would contradict the result of Eq. (34), which is derived from the spherical-symmetry hypothesis. For hypothesis (28) to be valid it is necessary that

$$
\frac{h}{2} \frac{\partial}{\partial z}\left\langle\Lambda_{1}^{2}\right\rangle=\left\langle\Lambda_{2}^{2}-\Lambda_{1}^{2}\right\rangle \ll\left\langle\Lambda^{2}\right\rangle,
$$

or

$$
\frac{\partial}{\partial z}\left\langle\Lambda^{2}\right\rangle \ll \frac{1}{h}\left\langle\Lambda^{2}\right\rangle .
$$

From Eq. (34) we have

$$
\begin{aligned}
\frac{h}{2} \frac{\partial\left\langle\Lambda^{2}\right\rangle}{\partial z} /\left\langle\Lambda^{2}\right\rangle & =\frac{h}{2 \Gamma} \frac{\exp (z / \Gamma)-1}{\exp (z / \Gamma)-1-z / \Gamma} \\
& \sim \begin{cases}h / z, & h \ll z \ll \Gamma \\
h /(2 \Gamma) \mathcal{O}(1), & z \simeq \Gamma \text { or } z \gg \Gamma\end{cases}
\end{aligned}
$$

In either case, inequality (39) is satisfied. A similar argument applies to other components. Therefore quasispherical hypothesis (28) is indeed a close approximation under the condition of long distance and weak PDL. Further confirmation is provided by numerically simulated statistics of $\Omega_{1,2,3}^{2}$ and $\Lambda_{1,2,3}^{2}$ in the rotating frame. Figures 4 and 5 confirm that the quasi-spherical symmetry postulation is indeed valid. 


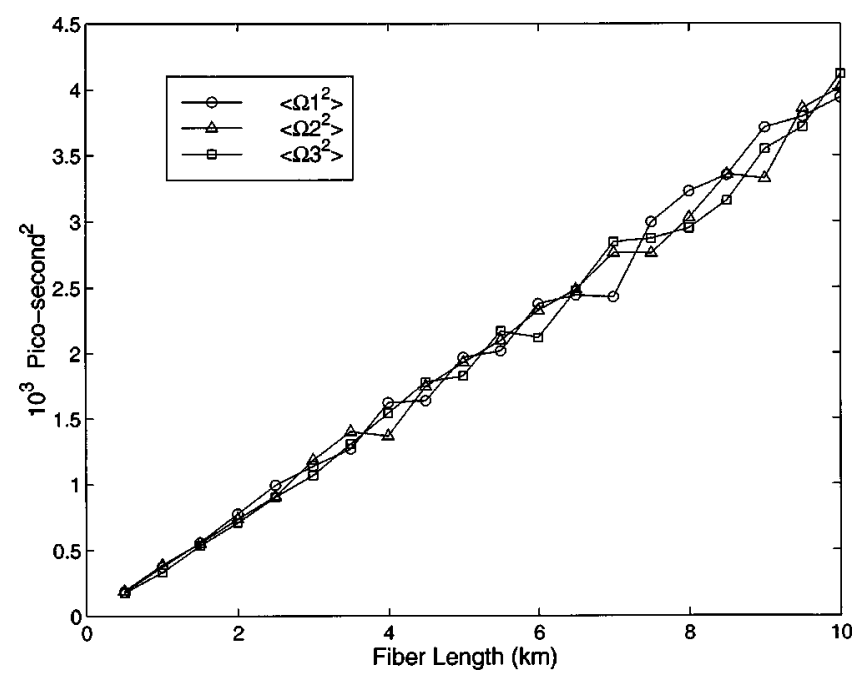

Fig. 4. Simulation of $\left\langle\Omega_{1,2,3}^{2}\right\rangle$ in the rotating coordinate system. Simulation parameters: $h=20(\mathrm{~m}), \alpha=10^{-3}\left(\mathrm{~m}^{-1}\right)$, and $\beta$ $=1.67 \times 10^{-13}\left(\mathrm{sec} \mathrm{m}^{-1}\right)$.

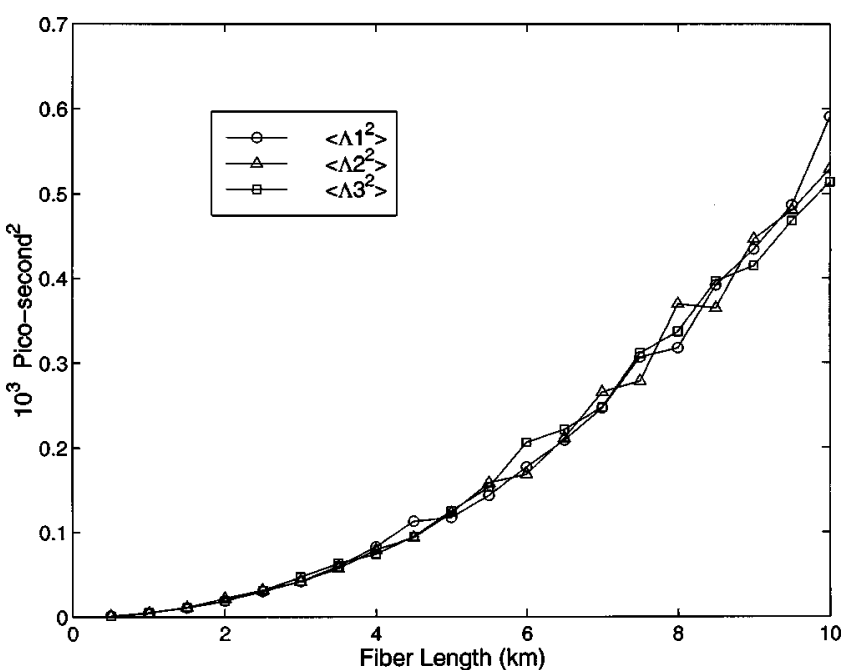

Fig. 5. Simulation of $\left\langle\Lambda_{1,2,3}^{2}\right\rangle$ in the rotating coordinate system. Simulation parameters: $h=20(\mathrm{~m}), \alpha=10^{-3}\left(\mathrm{~m}^{-1}\right)$, and $\beta$ $=1.67 \times 10^{-13}\left(\mathrm{sec} \mathrm{m}^{-1}\right)$.

As a practical example, we use the results obtained in Section 3 to calculate the statistics of the differential group delay (DGD) for a system suffering both PMD and PDL. Going back to state-vector evolution equation (6), we note that the generalized principal states are the eigenvectors of $T_{\omega} T^{-1}$, and the DGD of the system is given by the imaginary part of the difference of its two eigenvalues. $^{8}$ Owing to the tracelessness of $T_{\omega} T^{-1}$, its two eigenvalues can be written as $\pm(\eta+i \tau) / 2$. By definition, the DGD of the system is given by $\tau$. Taking the determinant on both sides of Eq. (3) and using the iden$\operatorname{tity} \operatorname{det}(\boldsymbol{\sigma} \cdot \mathbf{W})=-\mathbf{W}^{2}$, we obtain

$$
\tau^{2}=\frac{\boldsymbol{\Omega}^{2}-\Lambda^{2}}{2}+\sqrt{\frac{\left(\boldsymbol{\Omega}^{2}-\Lambda^{2}\right)^{2}}{4}+(\boldsymbol{\Omega} \cdot \boldsymbol{\Lambda})^{2}} .
$$

The dependence of $\tau^{2}$ on $\boldsymbol{\Omega}$ and $\Lambda$ is somewhat compli- cated but can be simplified in some cases. First we note from Eq. (25) and (37) that for $z \gg h$,

$$
\frac{(\boldsymbol{\Omega} \cdot \boldsymbol{\Lambda})^{2}}{\left(\mathbf{\Omega}^{2}-\Lambda^{2}\right)^{2}} \sim \begin{cases}\frac{1}{36} \frac{z}{\Gamma} \ll 1, & z \ll \Gamma \\ \mathcal{O}(1), & z \sim \Gamma . \\ \frac{\Gamma^{2}}{6 z^{2}} \exp \left(\frac{z}{\Gamma}\right) \gg 1, & z \gg \Gamma\end{cases}
$$

Then for $z \ll \Gamma$ we have

$$
\begin{aligned}
\left\langle\tau^{2}\right\rangle & \simeq\left\langle\boldsymbol{\Omega}^{2}-\boldsymbol{\Lambda}^{2}\right\rangle+\left\langle\frac{(\boldsymbol{\Omega} \cdot \boldsymbol{\Lambda})^{2}}{\boldsymbol{\Omega}^{2}-\boldsymbol{\Lambda}^{2}}\right\rangle \\
& \simeq\left\langle\boldsymbol{\Omega}^{2}-\boldsymbol{\Lambda}^{2}\right\rangle+\frac{\left\langle(\boldsymbol{\Omega} \cdot \boldsymbol{\Lambda})^{2}\right\rangle}{\left\langle\boldsymbol{\Omega}^{2}-\boldsymbol{\Lambda}^{2}\right\rangle}, \quad z \ll \Gamma .
\end{aligned}
$$

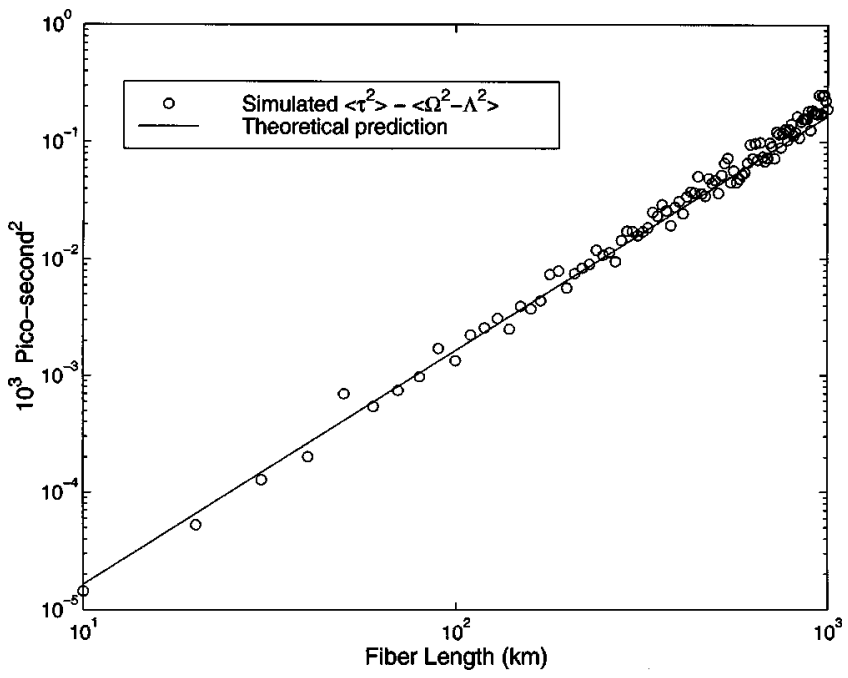

Fig. 6. Simulation of PDL effect on the mean-square DGD for $z \ll \Gamma$. The solid line is a theoretical prediction of Eq. (44), and discrete circles are numerical data. The numerical value of $\Gamma$ for the simulation is $1875(\mathrm{~km})$.

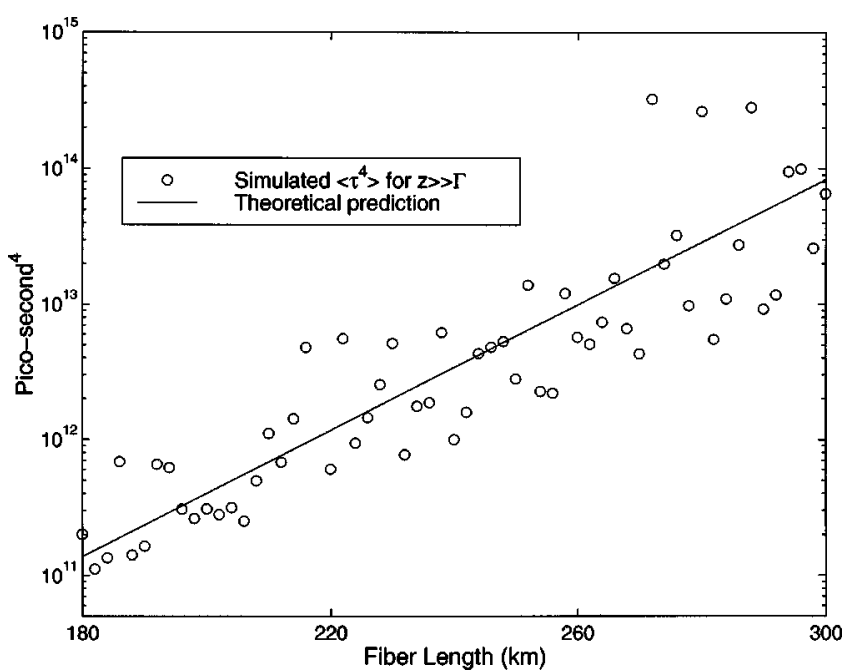

Fig. 7. Simulation of $\left\langle\tau^{4}\right\rangle$ for $z \gg \Gamma$. The solid line is a theoretical prediction of Eq. (45), and circles are numerical data. The $\Gamma$ value for this simulation is $20(\mathrm{~km})$. 
In the second step above, we have replaced the average of the ratio by the ratio of the average values for the purpose of a rough estimation. It follows from Eqs. (37) and (25) that

$$
\left\langle\tau^{2}\right\rangle-\left\langle\boldsymbol{\Omega}^{2}-\boldsymbol{\Lambda}^{2}\right\rangle \simeq \frac{\beta^{2} h}{18 \Gamma} z^{2}, \quad z \ll \Gamma .
$$

Figure 6 shows the agreement between the theoretical prediction of Eq. (44) and numerical results. As remarked in Section $3,\left\langle\boldsymbol{\Omega}^{2}-\boldsymbol{\Lambda}^{2}\right\rangle$ is exactly the meansquare PMD for the pure PMD case. Therefore we conclude that the deviation of $\left\langle\tau^{2}\right\rangle$ from its pure PMD value is $\left\langle\tau^{2}\right\rangle-\left\langle\tau^{2}\right\rangle_{\mathrm{PMD}} \sim z^{2}$ for $z \ll \Gamma$.

In the other extreme case of $z \gg \Gamma$, Eq. (41) reduces to

$$
\left\langle\tau^{4}\right\rangle \simeq\left\langle(\boldsymbol{\Omega} \cdot \boldsymbol{\Lambda})^{2}\right\rangle, \quad z \gg \Gamma .
$$

Although this situation is rarely encountered in real optical communications, it reveals an interesting phenomenon. Since $\left\langle(\boldsymbol{\Omega} \cdot \boldsymbol{\Lambda})^{2}\right\rangle$ scales exponentially with $z$ in this length regime, the average DGD also grows exponentially. If $z$ is large enough, $\langle\tau\rangle$ eventually becomes larger, and in fact can be much larger, than the sum of the local DGD (i.e., $\beta z$ in our continuous fiber model). This anomalously large global DGD was first observed in some particular discrete systems by Gisin and Huttner. ${ }^{8,9}$ Our analysis shows that this anomaly also exists in continuous systems, and once the length of the system is large enough, it is a universal phenomenon. Numerical simulation is performed to verify the exponential growth of the DGD in the regime of $z \gg \Gamma$. The numerical results are found to be consistent with the theoretical prediction (Fig. 7).

\section{ACKNOWLEDGMENT}

The financial support of the Office of Naval Research and that of the Air Force Office of Scientific Research are gratefully acknowledged.

\section{REFERENCES}

1. G. Foschini and C. Poole, "Statistical theory of polarization dispersion in single mode fibers," J. Lightwave Technol. 9, 1439-1456 (1991).

2. N. Gisin, "Solutions of the dynamical equation for polarization dispersion," Opt. Commun. 86, 371-373 (1991).
3. P. Wai and C. Menyuk, "Polarization mode dispersion, decorrelation, and diffusion in optical fibers with randomly varying birefringence," J. Lightwave Technol. 14, 148-157 (1996).

4. P. Ciprut, B. Gisin, N. Gisin, R. Passy, J. Von der Weid, F. Prieto, and C. Zimmer, "Second order polarization mode dispersion: impact on analog and digital transmissions," J. Lightwave Technol. 16, 757-771 (1998).

5. G. Foschini, R. Jopson, L. Nelson, and H. Kogelnik, "The statistics of PMD-induced chromatic fiber dispersion," J. Lightwave Technol. 17, 1560-1565 (1999).

6. M. Karlsson and J. Brentel, "Autocorrelation function of the polarization-mode dispersion vector," Opt. Lett. 24, 939941 (1999).

7. M. Shtaif, A. Mecozzi, and J. Nagel, "Mean-square magnitude of all orders of polarization mode dispersion and the relation with the bandwidth of the principal states," IEEE Photonics Technol. Lett. 12, 53-55 (2000).

8. N. Gisin and B. Huttner, "Combined effects of polarization mode dispersion and polarization dependent losses in optical fibers," Opt. Commun. 142, 119-125 (1997).

9. B. Huttner and N. Gisin, "Anomalous pulse spreading in birefringent optical fibers with polarization-dependent losses," Opt. Lett. 22, 504-506 (1997).

10. B. Huttner, C. De Barros, B. Gisin, and N. Gisin, "Polarization-induced pulse spreading in birefringent optical fibers with zero differential group delay," Opt. Lett. 24, 370-372 (1999).

11. L. Chen, J. Cameron, and X. Bao, "Statistics of polarization mode dispersion in presence of the polarization dependent loss in single mode fibers," Opt. Commun. 169, 69-73 (1999).

12. A. Yariv, Optical Electronics in Modern Communications (Oxford University, New York, 1997).

13. P. Dirac, The Principles of Quantum Mechanics (Oxford University, New York, 1958).

14. L. Schiff, Quantum Mechanics (McGraw-Hill, New York, 1968).

15. G. B. Arfken and H. J. Weber, Mathematical Methods for Physicists, 4th ed. (Academic, San Diego, Calif., 1995).

16. A. Eyal and M. Tur, "A modified Poincare sphere technique for the determination of polarization mode dispersion in the presence of differential loss/gain," In Optical Fiber Communications Conference, Vol. 2 of 1998 OSA Technical Digest Series (Optical Society of America, Washington, D.C., 1998), pp. 340-341.

17. C. D. Poole and R. E. Wagner, "Phenomenological approach to polarization dispersion in long single-mode fibers," Electron. Lett. 22, 1029-1030 (1986).

18. W. Horsthemke and R. Lefever, Noise Induced Transitions (Springer, Berlin, 1984).

19. L. Arnold, Stochastic Differential Equations: Theory and Applications (Wiley, New York, 1974).

20. B. Øksendal, Stochastic Differential Equations: An Introduction with Applications, 5th ed. (Springer, Berlin, 1998). 